\title{
Exposure to Mycobacterium avium Decreases the Protective Effect of the DNA Vaccine pVAXhsp65 Against Mycobacterium tuberculosis-Induced Inflammation of the Pulmonary Parenchyma
}

\author{
D. R. Martins*, A. C. Pelizon*, S. F. G. Zorzella-Pezavento*, J. Seger*, R. R. Santos Junior†, \\ D. M. Fonseca†, L. A. Justulin Jrt, C. L. Silva† \& A. Sartori*
}

*Department of Microbiology and Immunology, Biosciences Institute, Univ Estadual Paulista (UNESP), Botucatu, São Paulo, Brazil; $\dagger$ Department of Biochemistry and Immunology, University of São Paulo (USP), Ribeirão Preto, São Paulo, Brazil; and \$nstitute of Biologic and Natural Science, Federal University of Triângulo Mineiro (UFMT), Uberaba, Minas Gerais, Brazil

Received 24 August 2010; Accepted in revised form 30 December 2010

Correspondence to: A. Sartori, Department of Microbiology and Immunology, Biosciences Institute, Univ Estadual Paulista (UNESP), Distrito de Rubião Júnior, $\mathrm{s} / \mathrm{n}$, Botucatu, São Paulo 18618-000, Brazil. E-mail: sartori@ibb. unesp.br

\begin{abstract}
This work investigated the effect of previous Mycobacterium avium exposure on the protective ability of the DNA vaccine pVAXhsp65 against inflammation in the pulmonary parenchyma. BALB/c mice were presensitized with heatkilled M. avium and then immunized with three doses of pVAXhsp65 prior to challenge with Mycobacterium tuberculosis. M. avium sensitization induced high levels of spontaneous IL-5 production that were concomitant with a positive delayed-type hypersensitivity reaction; antigen-specific IFN- $\gamma$ production was also observed upon splenocyte stimulation. Prior exposure to $M$. avium resulted in altered cytokine and antibody production induced by immunization with pVAXhsp65; instead of a Th1 response, vaccinated mice previously exposed to $M$. avium developed a strong Th2 response. This switch to a Th2 response coincided with the loss of the anti-inflammatory effect of pVAXhsp65 vaccination previously observed in the pulmonary parenchyma of mice infected with M. tuberculosis. These results suggest that exposure to environmental mycobacteria can modulate immune responses induced by mycobacterial vaccines other than bacillus Calmette-Guérin.
\end{abstract}

\section{Introduction}

Tuberculosis (TB), which is caused by Mycobacterium tuberculosis, is responsible for more than two million deaths each year $[1,2]$ and ranks among the 10 principal causes of death and disability worldwide [3]. The increasing prevalence of AIDS and TB coinfections combined with the emergence of TB strains resistant to all major chemotherapeutic drugs has emphasized the need for effective prophylactic agents. The only available TB vaccine, bacillus Calmette-Guérin (BCG), has been distributed since the 1920s, and more than three billion people have received this vaccine. Although large variation in vaccine efficacy exists, the major conclusions arising BCG vaccination studies are that BCG vaccination efficiently protects against leprosy [4] as well as against the most severe childhood manifestations of TB (milliary and men- ingeal forms) [5]. However, the vaccine's efficacy in preventing pulmonary $\mathrm{TB}$ is very limited. These conclusions are consistent with the 15 -years follow-up trial of BCG vaccination comprising 360,000 individuals carried out in Cingleput, which found that BCG offered no protection against pulmonary TB in adults [6].

Many hypotheses have been proposed to explain the poor efficacy of BCG vaccination against pulmonary TB. Factors directly related to the vaccine, including inappropriate administration and storage have been suggested [7]. After the first successful vaccinations, BCG cultures were distributed worldwide and propagated under different conditions in various laboratories for several decades. This process resulted in genotypic and phenotypic differences between the original BCG strain and the various BCG daughter strains as well as differences among the daughter strains themselves [8]. Additionally, a number 
of hypotheses have suggested deficits in the immune response induced by BCG. As various $\mathrm{T}$ cell subsets participate in the immune response against mycobacteria, the lack of effective stimulation of the required $\mathrm{T}$ cell populations, especially CD8 $\mathrm{T}$ cells, may explain the insufficient levels of immunity induced by the BCG vaccine $[9,10]$. Finally, the exposure of human populations in tropical regions to large quantities of environmental mycobacteria has been suggested to be the single most important factor for the low efficacy of BCG vaccination in these regions $[11,12]$. The development of a new vaccine strategy is considered essential for the control of TB $[13,14]$. However, vaccine development is challenging, as any successful vaccine must overcome the litany of issues and obstacles described earlier.

Our group has been working with a DNA vaccine constructed by inserting the gene encoding heat-shock protein 65 from Mycobacterium leprae (groEL/ML0317) into plasmid vectors. These constructs displayed both a prophylactic $[15,16]$ and therapeutic effect in a murine model of TB [17, 18]. This vaccine was also able to prime neonatal mice for a strong immune response at the adult stage [19].

The objective of this study was to evaluate whether previous contact with Mycobacterium avium could interfere with the protective effect of pVAXhsp65 vaccination against pulmonary parenchyma inflammation triggered by $M$. tuberculosis. BALB/c mice were intradermally sensitized with heat-killed $M$. avium and then immunized with pVAXhsp65 prior to a challenge with $M$. tuberculosis. $M$. avium presensitization modulated pVAXhsp65 vaccine efficacy in a deleterious way: the vaccine lost its protective anti-inflammatory effect against TB-induced inflammation of the pulmonary parenchyma.

\section{Material and methods}

Animals. Female BALB/c mice were bred in the Animal Facility of São Paulo State University (UNESP) at the Biosciences Institute and used between 4 and 6 weeks of age. The animals were fed a standard pellet diet and water ad libitum. All of the experimental animal protocols and animal manipulations were approved by the local ethics committee and carried out in compliance with the ethical guidelines adopted by Colégio Brasileiro de Experimentação Animal.

Plasmid DNA construction and purification. The pVAXhsp65 vaccine was derived from the $\mathrm{pVAX}$ vector that uses the CMV intron (Invitrogen ${ }^{\circledR}$, Carlsbad, CA, USA). The pVAX vector was digested with BamH I and Not I (Gibco BRL, Gaithersburg, MD, USA), and a 3.3$\mathrm{kb}$ fragment corresponding to the $M$. leprae hsp 65 gene was inserted. The empty pVAX vector was used as a control. DH5 $\alpha$ Escherichia coli transformed with the pVAX plasmid or the plasmid carrying the hsp65 gene
(pVAXhsp65) were cultured in liquid LB medium (Gibco BRL) containing kanamycin $(50 \mu \mathrm{g} / \mathrm{ml})$. The plasmids were purified using the Concert High Purity Maxiprep System (Gibco BRL). Plasmid concentrations were determined by spectrophotometry at $\lambda=260$ and $280 \mathrm{~nm}$ using the Gene Quant II apparatus (Pharmacia Biotech, Buckinghamshire, UK).

Immunization procedures. Groups of $\mathrm{BALB} / \mathrm{c}$ mice were injected intradermally at the base of the tail with different concentrations of heat-killed M. avium (ATCC - 27294) $\left(0.08 \times 10^{6}, 4 \times 10^{6}\right.$ and $\left.200 \times 10^{6}\right)$. Three weeks later, these animals were evaluated for specific anti- $M$. avium immunity or immunized with pVAXhsp65. The mice were immunized with three doses of pVAXhsp65 $(100 \mu \mathrm{g} / 100 \mu \mathrm{l})$ plus $25 \%$ saccharose injected into the quadriceps muscle. The DNAhsp65 immunizations were administered at intervals of 15 days. Control animals received salt solution (the vaccine diluent) or $\mathrm{pVAX}$ (the empty vector).

Delayed-type bypersensitivity reaction. The delayed-type hypersensitivity (DTH) reaction was evaluated using the footpad-swelling test as previously described [20]. Briefly, control or immunized mice were inoculated in one of the back footpads with $1 \times 10^{7}$ heat-killed $M$. avium suspended in $50 \mu \mathrm{l}$ PBS. Footpad thickness was measured just prior to and $72 \mathrm{~h}$ after antigen inoculation using a dial caliper (Mitutoyo, Tokyo, Japan). The results were expressed in $\mathrm{mm}$.

Quantification of anti-hsp65 antibodies. Serum samples were collected by retro-orbital bleeding 3 weeks after heat-killed $M$. avium inoculation or 3 weeks after the last DNA vaccination, and hsp65-specific antibody levels were evaluated by enzyme-linked immunosorbent assay (ELISA). Maxisorp plates (Nunc, Roskilde, Denmark) were coated with $0.1 \mathrm{ml}(0.5 \mu \mathrm{g} / \mathrm{ml})$ of recombinant hsp65 (rhsp65) in coating solution (14.3 $\mathrm{mM} \mathrm{Na}_{2} \mathrm{CO}_{3}$ and $10.3 \mathrm{mM} \mathrm{NaHCO}, \mathrm{pH} 9.6$ ), incubated at $4{ }^{\circ} \mathrm{C}$ overnight. The plates were then blocked with $10 \%$ foetal calf serum (FCS) in PBS for $1 \mathrm{~h}$ at $37^{\circ} \mathrm{C}$. Serum samples were diluted 1:10 and tested. After incubation for $2 \mathrm{~h}$ at $37^{\circ} \mathrm{C}$, anti-mouse IgG1 and IgG2a biotinylated conjugates (A85-1 and R19-15, respectively, from PharMingen, Fallbrook, CA, USA) were added for detection of specific isotype antibodies. After washing, the plates were incubated at room temperature for $30 \mathrm{~min}$ with the StreptAB kit (Dako, Carpinteria, CA, USA) and then developed by adding $\mathrm{H}_{2} \mathrm{O}_{2}$ and o-phenylenediamine dihydrochloride (OPD). Colour development was stopped with $\mathrm{H}_{2} \mathrm{SO}_{4}$, and the optical density was measured at $492 \mathrm{~nm}$.

Evaluation of cytokine production. Spleen cells were obtained 3 weeks after heat-killed $M$. avium inoculation or 3 weeks after the last vaccination dose. The cells were adjusted to a concentration of $5 \times 10^{6}$ cells $/ \mathrm{ml}$ in RPMI 1640 medium supplemented with $5 \%$ FCS, $20 \mathrm{~mm}$ glutamine and $40 \mathrm{IU} / \mathrm{l}$ of gentamicin. Next, the cells were 
cultured in 48-well, flat-bottomed culture plates (Nunc, Life Tech. Inc., Maryland, MA, USA) in the presence of heat-killed M. avium $\left(1.2 \times 10^{7} / \mathrm{ml}\right)$, rhsp65 $(10 \mu \mathrm{g} / \mathrm{ml})$ or concanavalin A (ConA) $(10 \mu \mathrm{g} / \mathrm{ml})$. Culture supernatants were harvested $48 \mathrm{~h}$ later, and cytokine levels were evaluated by ELISA. Cytokines were measured following manufacturer instructions (PharMingen). Purified monoclonal antibodies against IFN- $\gamma$ (R4-6A2) and IL-5 (TRKF5) were used at $1 \mu \mathrm{g} / \mathrm{ml}$ as capture antibodies. The following biotinylated antibodies were used for detection: anti-IFN- $\gamma$ (XMG1.2) and anti-IL-5 (TRFK4) at $0.5 \mu \mathrm{g} / \mathrm{ml}$. After an incubation step followed by washing, plates were incubated with the StreptAB kit (Dako) at room temperature for $30 \mathrm{~min}$ and then developed by adding $\mathrm{H}_{2} \mathrm{O}_{2}$ and OPD. Colour development was stopped with $\mathrm{H}_{2} \mathrm{SO}_{4}$, and the optical density was measured at $492 \mathrm{~nm}$. Cytokine concentrations were determined by comparison with standard curves obtained from recombinant cytokine samples run in duplicate.

Infection with $M$. tuberculosis and histopathological analysis. Groups of mice previously injected with heat-killed $M$. avium, vaccinated with pVAXhsp65 or mice that were exposed to $M$. avium and vaccinated were challenged 4 weeks later with $M$. tuberculosis (H37Rv - ATCC 27294). The animals were first anaesthetized with tribromoethanol 2.5\% (Acros Organics, Geel, Belgium) and then challenged through intratracheal instillation of a bacterial solution $\left(10^{4}\right.$ bacilli/animal). Four weeks later, the animals were euthanized, and histopathological analysis was performed on lung sections. The upper left lung lobe of each mouse was fixed in $10 \%$ formalin, embedded in Paraplast plus (McCormick Scientific, St. Louis, MO, USA), prepared routinely and then sectioned for light microscopy. Sections ( $5 \mu \mathrm{m}$ each) were stained with haematoxylin and eosin. Pneumonic areas were measured using the image analyzer Leica QWin software Version 3 for Windows ${ }^{\mathrm{TM}}$ (Microsoft Corporation, Redmond, WA, USA). Eight random fields for each experimental group were analysed at $200 \times$ magnification.

Statistical analysis. Results represent the mean \pm SEM for each variable. Statistical analysis was performed using GraphPad InStat Version 1993 (San Diego, CA, USA). The nonparametric Mann-Whitney test was used to compare cytokine and antibody levels. Values of $P<0.05$ were considered statistically significant.

\section{Results}

Inoculation with M. avium is associated with high levels of IL-5 production

Three weeks after injection with heat-killed M. avium, animals were challenged with particulate $M$. avium antigen, and the increase in the footpad thickness was measured $72 \mathrm{~h}$ later. A significant increase in footpad size was observed in the group inoculated with the higher dose of heat-killed $M$. avium compared to the non-immunized group (Fig. 1A).

Immediately following footpad measurements, the animals were euthanized, and their spleens were harvested for analysis. Splenocyte cell cultures were stimulated with $M$. avium or ConA, and IFN- $\gamma$ and IL-5 concentrations were evaluated in the culture supernatants. Significant antigen-specific IFN- $\gamma$ induction was detected in cell culture supernatants from mice previously inoculated with the intermediate and higher doses of heat-killed M. avium. Although all of the experimental groups exhibited significant IFN- $\gamma$ production in response to ConA stimulation, IFN- $\gamma$ levels were significantly lower in the three groups that were previously inoculated with M. avium (Fig. 1B). Stimulation with ConA-induced similar IL-5 levels in all of the experimental groups. Strikingly, splenic cells from mice exposed to $M$. avium spontaneously produced significant levels of IL-5; endogenous IL-5 production was most apparent in cell cultures from mice that received either the intermediate or high dose of heat-killed M. avium (Fig. 1C). Antigen-specific stimulation did not increase IL-5 production, and they were similar to the ones induced by polyclonal stimulation with ConA.

\section{M. avium exposure modulates the immune response induced by DNAhsp65 vaccination}

Three weeks after being injected with different $M$. avium doses, BALB/c mice were immunized with pVAXhsp65. Control groups received saline or empty vector (pVAX). Two weeks after the last dose, the mice were euthanized and splenic cytokine production and hsp65-specific antibody levels were examined. Prior exposure to heat-killed $M$. avium decreased both rhsp65-specific and ConAinduced IFN- $\gamma$ production (Fig. 2A). Unstimulated, or endogenous, production of IL-5 was significantly higher in mice that were inoculated with the intermediate and the higher doses of $M$. avium. In response to ConA stimulation, pVAXhsp65-vaccinated mice produced less IL-5 than the control group. Interestingly, the previous contact with intermediate or high amounts of $M$. avium abolished the downmodulatory effect of pVAXhsp65 vaccination on IL-5 production (Fig. 2B). M. avium exposure also affected antibody production, increasing both IgG1and IgG2a-specific antibodies: this increase was highly significant in animals inoculated with the higher dose of M. avium (Fig. 2C).

\section{M. avium exposure decreases the protective effect of DNAhsp65 over the pulmonary parenchyma}

Immunization with 3 doses of pVAXhsp65 resulted in protection of the pulmonary parenchyma against $M$. tuberculosis-induced inflammation. However, this effect was 
A

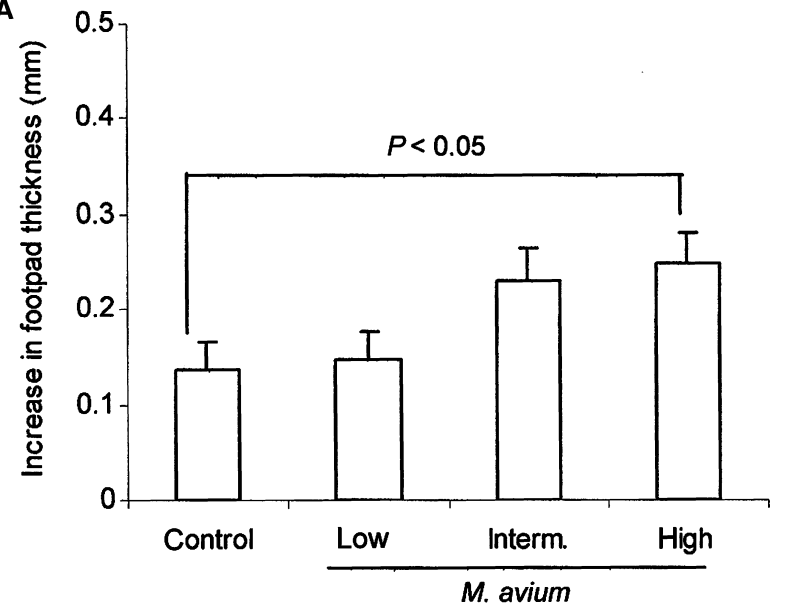

B

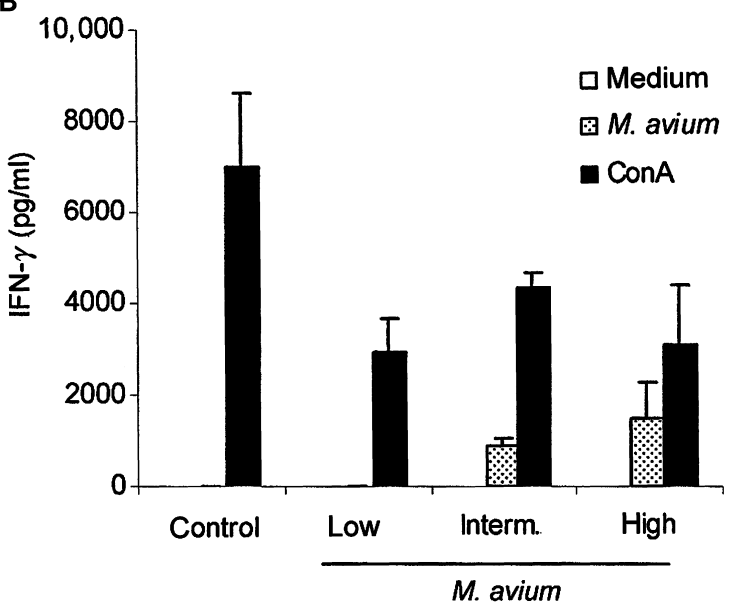

C

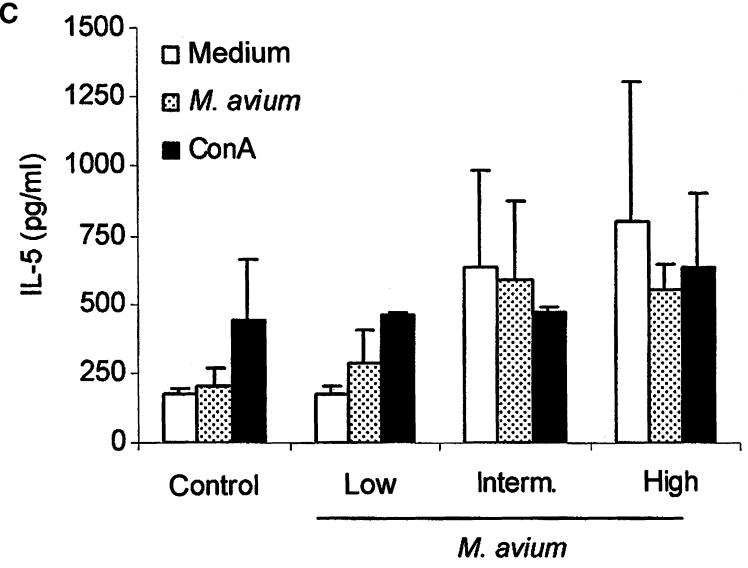

Figure 1 Immune response induced by injection with heat-killed Mycobacterium avium. BALB/c mice were injected with different concentrations of heat-killed M. avium $\left(0.08 \times 10^{6}, 4 \times 10^{6}\right.$ and $200 \times$ $10^{6} \mathrm{CFU}$ ) by the subcutaneous route at the base of tail. Delayed-type hypersensitivity (DTH) (A) and in vitro cytokine production were evaluated 3 weeks later. DTH was tested by inoculation of $M$. avium $\left(10^{7} \mathrm{CFU} / 50 \mu \mathrm{l}\right)$ in the left footpad. Footpad thickness was evaluated after $72 \mathrm{~h}$ using a caliper. IFN- $\gamma$ (B) and IL-5 (C) were quantified by enzyme-linked immunosorbent assay in spleen cell cultures stimulated with M. avium $\left(10^{7} \mathrm{CFU} / \mathrm{ml}\right)$ or ConA $(10 \mu \mathrm{g} / \mathrm{ml})$ for $48 \mathrm{~h}$. The results represent the geometric mean \pm SEM of three animals. blocked by previous exposure to $M$. avium. Lung sections from control mice showing a preserved pulmonary parenchyma can be observed in Fig. 3A. As expected, M. tuberculosis infection elicited the appearance of small- and medium-sized focal granulomas characterized by perivascular and peribronchial mononuclear cell infiltration. Necrotic areas were also observed throughout the lung tissue (Fig. 3B). Lung sections from mice immunized with pVAXhsp65 prior to infection with $M$. tuberculosis exhibited tissue architecture very similar to lung sections from control, untreated mice; only a few inflammatory foci were observed (Fig. 3C). However, lung sections from mice that were injected with $M$. avium before $M$. tuberculosis infection showed intense peribronchial and perivascular inflammatory infiltrates. Necrotic areas were also observed (Fig. 3D). Thus, M. avium exposure prior to pVAXhsp65 immunization completely abolished the protective effect of pVAXhsp65 over the pulmonary parenchyma (Fig. 3E). The loss of pVAXhsp65 protection was further confirmed by a quantitative comparison of the percentage of pneumonic areas in each of the different treatment groups (Fig. 3F).

\section{Discussion}

Mycobacterium bovis BCG, the current vaccine against infection with $M$. tuberculosis, offers variable protective efficacy in man. It has been suggested that exposure to environmental mycobacteria can interfere with the generation of BCG-specific immunity. pVAXhsp65 is a genetic construct containing the bsp65 gene from $M$. leprae that has demonstrated prophylactic and therapeutic effects in experimental TB models; it is currently being optimized in numerous preclinical studies. As hsp65 is a ubiquitous antigen present in many bacteria, including all Mycobacterium sp., we asked whether previous exposure to these bacteria could affect the immunogenicity and protective efficacy of this vaccine, similar to the described effect of mycobacteria exposure on BCG vaccine efficacy.

Theoretically, subunit vaccines devised to substitute BCG would be less affected by environmental mycobacteria. This hypothesis has been based on the supposition that BCG vaccination failure is caused by cross-protective immunity, generated from exposure to environmental mycobacteria, that drives the early clearance of BCG. However, this report provides evidence that previous exposure to $M$. avium affects both the immunogenicity and protective effect of pVAXhsp65 vaccination.

We began our study by evaluating the immunogenicity of a preparation of heat-killed $M$. avium. Adult $\mathrm{BALB} / \mathrm{c}$ mice were injected at the base of the tail following procedures already adopted by other authors [21]. Three different doses of heat-killed $M$. avium were tested: $0.08 \times 10^{6}$ (low), $4 \times 10^{6}$ (intermediate) and $200 \times 10^{6}$ (high). Three weeks later, mice exposed to heat-killed 

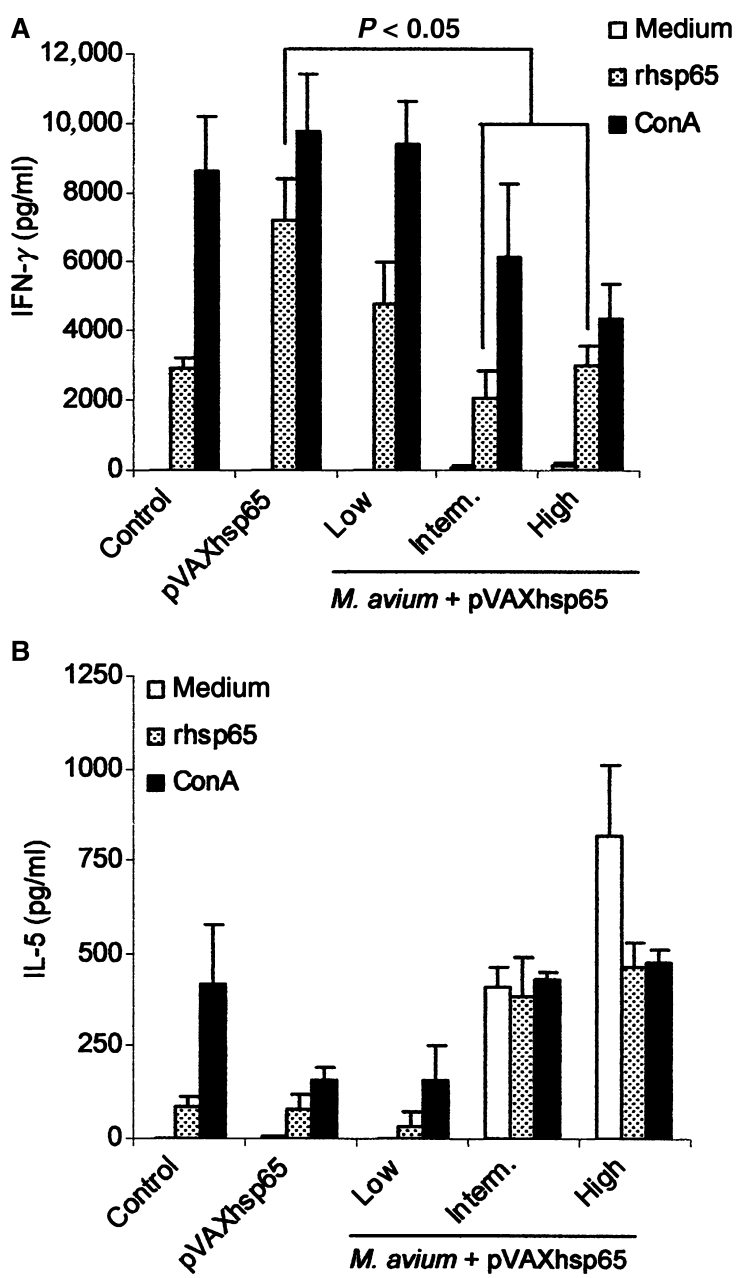

C

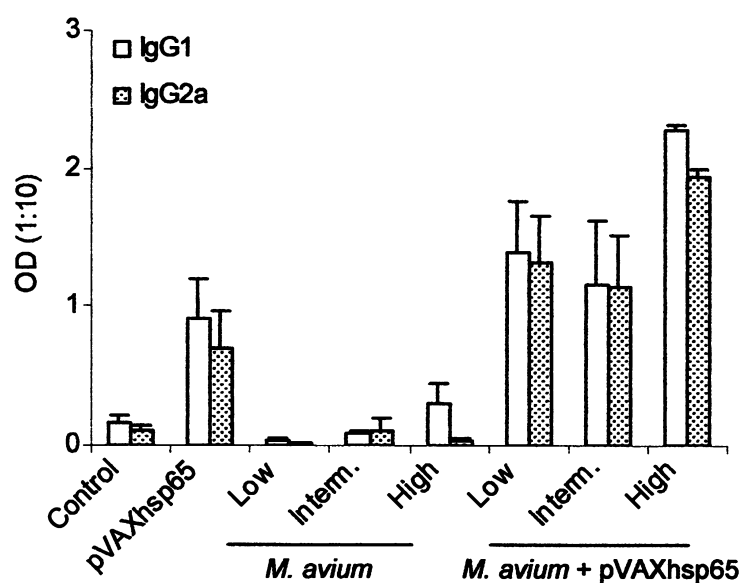

Figure 2 Effect of previous contact with Mycobacterium avium on the immune response induced by pVAXhsp65 immunization. BALB/c mice were injected with heat-killed M. avium $\left(0.08 \times 10^{6}, 4 \times 10^{6}\right.$ and $200 \times 10^{6}$ CFU) by the subcutaneous route at the base of the tail. Three weeks later, they were immunized with three doses of pVAXhsp65 (100 $\mu \mathrm{g}$ each). The doses were administered 15 days apart. Two weeks after the last pVAXhsp65 dose, anti-hsp65 levels (A) were evaluated in blood samples and IFN- $\gamma$ (B) and IL-5 (C) production were evaluated in spleen cell cultures by enzyme-linked immunosorbent assay. The results represent the geometric mean \pm SEM of five animals.
M. avium exhibited a significant DTH reaction and stimulations of splenocyte cultures showed $M$. avium-specific production of IFN- $\gamma$ induction. However, contrasting with these results, a significant decrease in IFN- $\gamma$ production was observed in ConA-stimulated splenocyte cultures from mice immunized with heat-killed M. avium. Concomitantly, striking endogenous IL-5 production was detected in splenocyte cultures from mice exposed to $M$. avium. Levels of IL-5 production correlated to the dosage of $M$. avium injected; IL-5 levels increased with increased antigen exposure. A literature report supports our IL-5 finding. Coussens et al. [22], by using microarray analysis, suggested that $\mathrm{T}$ cells within the peripheral blood mononuclear cell (PBMC) population from M. avium subspecies paratuberculosis (M. paratuberculosis)infected cows were skewed towards a Th2-like phenotype with the high expression of genes encoding IL-5 and GATA-3, a Th2-polarizing transcription factor. Therefore, we could attribute the decreased IFN- $\gamma$ production in ConA-stimulated cultures to a Th2-polarized response.

However, this scenario seems to fit better with other characteristics of the $M$. avium complex. This group of mycobacteria is distinguished by the presence of a unique, highly antigenic group of lipids located on the cell surface known as glycopeptidolipids (GPLs) [23]. These molecules have been shown to be immunosuppressive and can induce a variety of immunomodulatory cytokines [24]. The total lipids in M. avium significantly suppressed the secretion of IL-12 and IFN- $\gamma$ that are associated with a Th1 response $[25,26]$. However, many other mechanisms must also be considered as the main or secondary causes of the decreased IFN- $\gamma$ production. For example, during $M$. avium infection, $\mathrm{T}$ cells upregulate expression of $\mathrm{CD} 95$ on their surface and can be stimulated to undergo apoptosis by cross-linking of Fas [27, 28]. Additionally, it has been demonstrated that mycobacterial GPLs become inserted into phospholipid monolayers, disrupting in this way the responsiveness of different cell types, including mononuclear cells [29].

To test whether previous contact with $M$. avium could affect the immune response induced by pVAXhsp65 vaccination, mice were injected with heat-killed $M$. avium, and 3 weeks later, they were immunized with pVAXhsp65. Two parameters were evaluated, vaccine immunogenicity and protection against lung injury. Immunogenicity was ascertained by rhsp65-specific production of IFN- $\gamma$ and IL- 5 and by the levels of anti-hsp65 antibodies in the serum. Pulmonary injury was determined by the quantification of pneumonic areas in the lungs.

In terms of cytokine production, vaccination with pVAXhsp65 induced a predominantly Th1 profile characterized by high levels of IFN- $\gamma$ and only basal IL- 5 levels. These results are comparable with previous publications utilizing pVAXhsp65 or other DNA vaccines [30, 31]. A clear immunomodulatory effect was triggered by previous 

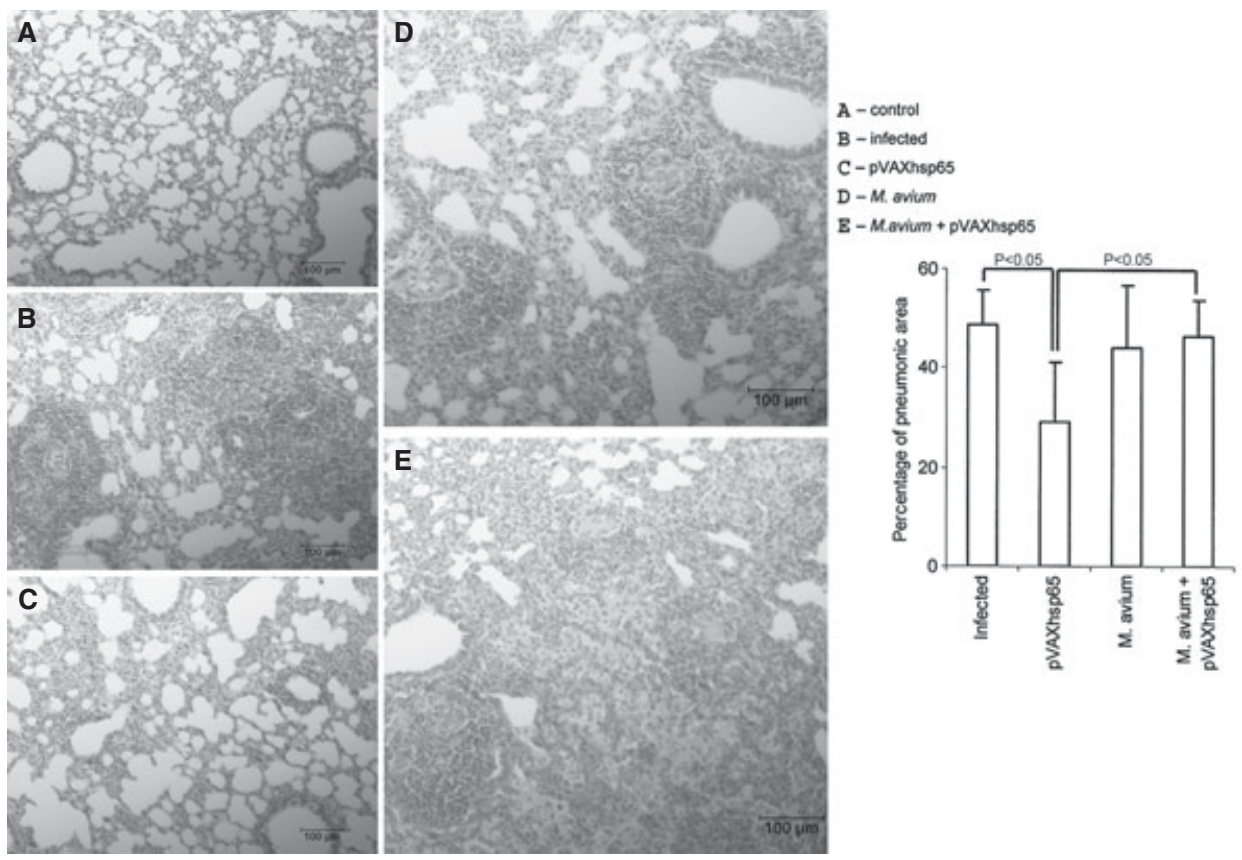

Figure 3 Effect of previous contact with Mycobacterium avium on the protective ability of pVAXhsp65 vaccination over the pulmonary parenchyma. $\mathrm{BALB} / \mathrm{c}$ mice were injected with $200 \times 10^{6} \mathrm{CFU}$ of heat-killed $M$. avium by an intradermal route at the base of the tail and then immunized with 3 doses of pVAXhsp65. Two weeks later, the mice were infected with Mycobacterium tuberculosis ( $10^{4}$ bacilli/animal) by an intratracheal route. A quantitative histopathological analysis of the pneumonic areas was performed 30 days after infection. Lung samples from control (A) and mice infected with $M$. tuberculosis (B-E). Before infection, the animals were submitted to no treatment (B), to immunization with pVAXhsp65 (C), to inoculation with heat-killed M. avium (D) and to inoculation with M. avium and immunization with pVAXhsp65 (E). A-E are representative of five animals analysed. A quantitative comparison of the pneumonic areas among the four infected groups is shown in (F). This figure represents the mean of five animals analysed in each group.

contact with $M$. avium. Prior exposure to $M$. avium resulted in the pronounced reduction in rhsp65-specific and ConA-induced IFN- $\gamma$ production; this result was most prominent with the high dose of $M$. avium. Interestingly, prior exposure to $M$. avium altered pVAXhsp65 vaccination-induced IL-5 responses; pVAXhsp65 vaccination was unable to downmodulate IL-5 production in mice previously exposed to $M$. avium. To obtain a clearer picture of this data, it was transformed into numerical values by calculating the ratio of IFN- $\gamma$ to IL- 5 levels in the pVAXhsp65 and M. avium/pVAXhsp65 experimental groups. This ratio dropped from 1685 in the first group to 6 in the second one, confirming that exposure to $M$. avium clearly changed the cytokine profile induced by pVAXhsp65 vaccination.

Even though $M$. avium exposure alone did not induce anti-hsp65 antibodies (data not shown), it significantly increased both hsp65-specific IgG1 and IgG2a antibody levels induced by pVAXhsp65 vaccination. The lack of anti-hsp65 antibodies in mice injected with heat-killed M. avium was a little unexpected because hsp65 has been described as an immunodominant antigen in mycobacterial infections [32]. However, in work by Nagabhushanam et al. [33], hsp65 was not considered a dominant $\mathrm{T}$ cell antigen in $M$. avium infections.
Thus, M. avium priming shaped pVAXhsp65-induced immunity such that a predominantly Th1 profile turned into a predominantly Th2 profile accompanied by a striking increase in antigen-specific antibody production. In the context of TB vaccines, a non-protective effect was already expected. Protection against TB appears to require not only a strong Th1 profile $[34,35]$ but also a concomitant immunoregulation that downmodulates Th2 response [36, 37].

Previous work from our laboratory has indicated a close relationship between the ability of pVAXhsp65 vaccination to decrease bacterial load and its ability to protect the pulmonary parenchyma from injury caused by $M$. tuberculosis $[16,18]$. Here, we analysed the effect of previous exposure to $M$. avium on the ability of pVAXhsp65 vaccination to prevent lung injury by comparing the percentages of pneumonic areas. Lung sections from mice immunized with pVAXhsp65 prior to M. tuberculosis infection presented a structure very similar to control mice, with the presence of a limited number of inflammatory foci. Exposure to $M$. avium prior to infection with $M$. tuberculosis had no protective effect; further $M$. avium exposure prior to pVAXhsp65 vaccination completely abolished the protective effect pVAXhsp65 vaccination upon challenge with $M$. tuberculosis. 
From a broad point of view, our results are supported by the literature that generally shows the deleterious interference of environmental mycobacteria in BCG vaccine efficacy [38-40]. Experiments performed using the $M$. avium complex have recently demonstrated that presensitization with species from this complex can modify BCG-induced immunity [41, 42]. In 2006, Flaherty et al. [43] published a well-conducted study examining exposure to environmental mycobacteria following BCG vaccination. These authors demonstrated that exposure to $M$. avium by the gastrointestinal route interfered with the established protective immune response generated by the previous BCG vaccination.

To explain our results in this context, we could imagine that previous contact with $M$. avium-primed the immune response against the hsp65 antigen. This hypothesis is plausible as hsp65 is highly conserved and present in all Mycobacterium spp. despite not being very immunogenic in $M$. avium [33]. The significant increase in IgG1 and IgG2a anti-hsp65 antibodies observed after pVAXhsp65 immunization in $M$. avium-primed mice supports this possibility. Additionally, it is possible that the high content of GPLs in the heat-killed $M$. avium preparation could be more available because of the heatkilling procedure, which would have a Th2-polarizing effect.

Possible priming with $M$. avium hsp65 could also be analysed from the perspective of the 'original sin' concept. This hypothesis was proposed by Francis [44] in the context of serological data from influenza infections. Later on, it was extended to cellular immunity and dengue infections [45]. According to the more encompassing theory, the immune response to a current infection may be dominated by $\mathrm{T}$ cells or antibodies that have higher avidity for epitopes encountered from a previous pathogen [46]. In this context, we could imagine that $M$. avium hsp65 primed the immune system, stamping an 'antigenic sin' that was later expanded by pVAXhsp65 vaccination. This enhanced anti-hsp65 response was not, however, protective in this model as it was highly polarized to a Th2-type response. If this reasoning is correct, we could hypothesize that a Th1polarizing effect during the first encounter with hsp65 would allow a protective type of response to develop later.

In summary, we demonstrated that exposure to $M$. avium increased humoral responses but decreased cellular immunity induced by pVAXhsp65 vaccination. Also, these changes abolished the ability of pVAXhsp65 vaccination to protect the pulmonary parenchyma against $M$. tuberculosis-induced injury. These findings have particular relevance in the search for a new tuberculosis vaccine, as the areas where the vaccine is urgently required are the areas with a higher predominance of environmental mycobacteria.

\section{Acknowledgment}

The authors are grateful to Fundação de Amparo à Pesquisa do Estado de São Paulo (FAPESP) that provided grant funding for this study.

\section{Conflict of interest}

The authors declare no conflicts of interest.

\section{References}

1 Rook GA, Dheda K, Zumla A. Immune responses to tuberculosis in developing countries: implications for new vaccines. Nat Rev Immunol 2005;5:661-7.

2 Dye C, Lönnroth K, Jaramillo E, Williams BG, Raviglione M. Trends in tuberculosis incidence and their determinants in 134 countries. Bull World Health Org 2009;87:683-91.

3 Korenromp EL, Bierrenbach AL, Williams BG, Dye C. The measurement and estimation of tuberculosis mortality. Int J Tuberc Lung Dis 2009;13:283-303.

4 Velema JP, Ogbeiwi OI. ILEP organisations should strive for high BCG coverage in communities at risk of leprosy. Lepr Rev 2007;78:88-101.

5 Rodrigues LC, Diwan VK, Wheeler JG. Protective effect of BCG against tuberculous meningitis and miliary tuberculosis: a metaanalysis. Int J Epidemiol 1993;22:1154-8.

6 Tuberculosis Research Centre (ICMR), Chennai. Fifteen year follow up of trial of BCG vaccines in south India for tuberculosis prevention. Indian J Med Res 1999;110:56-69.

7 Fine PE. Variation in protection by BCG: implications of and for heterologous immunity. Lancet 1995;346:1339-45.

8 Behr MA. BCG-different strains, different vaccines? Lancet Infect Dis 2002;2:86-92.

9 Hess J, Kaufmann SH. Development of novel tuberculosis vaccines. C R Acad Sci III 1999;322:953-8.

10 Behar SM, Woodworth JS, Wu Y. Next generation: tuberculosis vaccines that elicit protective CD8+ T cells. Expert Rev Vaccines 2007;6:441-56.

11 Palmer CE, Long MW. Effects of infection with atypical mycobacteria on BCG vaccination and tuberculosis. Am Rev Respir Dis 1966;94:553-68.

12 McShane H. Vaccine strategies against tuberculosis. Swiss Med Wkly 2009;139:156-60.

13 Hernàndez-Pando $\mathrm{R}$, Castañòn $\mathrm{M}$, Espitia $\mathrm{C}$, Lopez-Vidal $\mathrm{Y}$. Recombinant BCG vaccine candidates. Curr Mol Med 2007;7:36572.

14 Kaufmann SH. Rational design of novel antibacterial vaccines with an emphasis on tuberculosis. Scand J Infect Dis 2008;40:595-600.

15 Bonato VL, Lima VM, Tascon RE, Lowrie DB, Silva CL. Identification and characterization of protective $T$ cells in hsp65 DNA-vaccinated and Mycobacterium tuberculosis-infected mice. Infect Immun 1998;66:169-75.

16 de Paula L, Silva CL, Carlos D et al. Comparison of different delivery systems of DNA vaccination for the induction of protection against tuberculosis in mice and guinea pigs. Genet Vaccines Ther 2007;5:2.

17 Lowrie DB, Tascon RE, Bonato VL et al. Therapy of tuberculosis in mice by DNA vaccination. Nature 1999;400:269-71.

18 Bonato VL, Gonçalves ED, Soares EG et al. Immune regulatory effect of pHSP65 DNA therapy in pulmonary tuberculosis: activation of CD8 + cells, interferon-gamma recovery and reduction of lung injury. Immunology 2004;113:130-8. 
19 Pelizon AC, Martins DR, Zorzella SFG et al. Genetic vaccine for tuberculosis (pVAXhsp65) primes neonate mice for a strong immune response at the adult stage. Genet Vaccines Ther 2007; $5: 12$.

20 Fazioli RA, Singer-Vermes LM, Kashino SS et al. Delayed-type hypersensitivity response in an isogenic murine model of paracoccidioidomycosis. Mycopathologia 1994;126:137-46.

21 Hernandez-Pando R, Pavön L, Arriaga K, Orozco H, Madrid-Marina V, Rook G. Pathogenesis of tuberculosis in mice exposed to low and high doses of an environmental mycobacterial saprophyte before infection. Infect Immun 1997;65:3317-27.

22 Coussens PM, Pudrith CB, Skovgaard K et al. Johne's disease in cattle is associated with enhanced expression of genes encoding IL-5, GATA-3, tissue inhibitors of matrix metalloproteinases 1 and 2, and factors promoting apoptosis in peripheral blood mononuclear cells. Vet Immunol Immunopathol 2005;15:221-34.

23 Schorey JS, Sweet L. The mycobacterial glycopeptidolipids: structure, function, and their role in pathogenesis. Glycobiology 2008; 18:832-41.

24 Chatterjee D, Khoo KH. The surface glycopeptidolipids of mycobacteria: structures and biological properties. Cell Mol Life Sci 2001;58:2018-42.

25 Horgen L, Barrow EL, Barrow WW, Rastogi N. Exposure of human peripheral blood mononuclear cells to total lipids and serovar-specific glycopeptidolipids from Mycobacterium avium serovars 4 and 8 results in inhibition of TH1-type responses. Microb Pathog 2000;29:9-16.

26 Pourshafie MR, Sonnenfeld G, Barrow WW. Immunological and ultrastructural disruptions of $\mathrm{T}$ lymphocytes following exposure to the glycopeptidolipid isolated from the Mycobacterium avium complex. Scand J Immunol 1999;49:405-10.

27 Zhong J, Gilbertson B, Cheers C. Apoptosis of CD4+ and CD8+ T cells during experimental infection with Mycobacterium avium is controlled by Fas/FasL and Bcl-2-sensitive pathways, respectively. Immunol Cell Biol 2003;81:480-6.

28 Roger PM, Bermudez LE. Infection of mice with Mycobacterium avium primes CD8+ lymphocytes for apoptosis upon exposure to macrophages. Clin Immunol 2001;99:378-86.

29 Vergne I, Prats M, Tocanne JF, Lanéelle G. Mycobacterial glycopeptidolipid interactions with membranes: a monolayer study. FEBS Lett 1995;375:254-8.

30 Silva CL, Bonato VL, Lima VM, Faccioli LH, Leão SC. Characterization of the memory/activated $\mathrm{T}$ cells that mediate the long-lived host response against tuberculosis after bacillus Calmette-Guérin or DNA vaccination. Immunology 1999;97:573-81.

31 Grover A, Ahmed MF, Singh B, Verma I, Sharma P, Khuller GK. A multivalent combination of experimental antituberculosis DNA vaccines based on $\mathrm{Ag} 85 \mathrm{~B}$ and regions of difference antigens. Microbes Infect 2006;8:2390-9.

32 van Eden W, van der Zee R, Prakken B. Heat-shock proteins induce t-cell regulation of chronic inflammation. Nat Rev Immunol 2005;5:319-30.

33 Nagabhushanam V, Praszkier J, Cheers C. Molecular and immunological characterization of Mycobacterium avium $65 \mathrm{kDa}$ heat shock protein (Hsp65). Immunol Cell Biol 2001;79:454-61.

34 Raja A. Immunology of tuberculosis. Indian $J$ Med Res 2004;120:213-32.

35 Fletcher HA. Correlates of immune protection from tuberculosis. Curr Mol Med 2007;7:319-25.

36 Rook GA, Dheda K, Zumla A. Do successful tuberculosis vaccines need to be immunoregulatory rather than merely Th1-boosting? Vaccine 2005;23:2115-20.

37 Wang J, Qie Y, Zhu B et al. Evaluation of a recombinant BCG expressing antigen Ag85B and PPE protein Rv3425 from DNA segment RD11 of Mycobacterium tuberculosis in C57BL/6 mice. Med Microbiol Immunol 2009;198:5-11.

38 Brandt L, Feino Cunha J, Weinreich Olsen A et al. Failure of the Mycobacterium bovis BCG vaccine: some species of environmental mycobacteria block multiplication of BCG and induction of protective immunity to tuberculosis. Infect Immun 2002;70:672-8.

39 Andersen P, Doherty TM. The success and failure of BCG - implications for a novel tuberculosis vaccine. Nat Rev Microbiol 2005;3:656-62.

40 Hoft DF. Tuberculosis vaccine development: goals, immunological design, and evaluation. Lancet 2008;372:164-75.

41 de Lisle GW, Wards BJ, Buddle BM, Collins DM. The efficacy of live tuberculosis vaccines after presensitization with Mycobacterium avium. Tuberculosis 2005;85:73-9.

42 Young SL, Slobbe L, Wilson R, Buddle BM, de Lisle GW, Buchan GS. Environmental strains of Mycobacterium avium interfere with immune responses associated with Mycobacterium bovis BCG vaccination. Infect Immun 2007;75:2833-40.

43 Flaherty DK, Vesosky B, Beamer GL, Stromberg P, Turner J. Exposure to Mycobacterium avium can modulate established immunity against Mycobacterium tuberculosis infection generated by Mycobacterium bovis BCG vaccination. J Leukoc Biol 2006;80:1262-71.

44 Francis T. On the doctrine of original antigenic sin. Proc Am Philos Soc 1960;104:572-8.

45 Mathew A, Rothman AL. Understanding the contribution of cellular immunity to dengue disease pathogenesis. Immunol Rev 2008;225:300-13.

46 Zehn D, Turner MJ, Lefrançois L, Bevan MJ. Lack of original antigenic sin in recall $\mathrm{CD} 8+\mathrm{T}$ Cell responses. $J$ Immunol 2010;184:6320-6. 